\title{
Development of a shortened FFQ to assess a 'prudent' dietary pattern amongst women in Southampton
}

\author{
Sarah Crozier, Hazel Inskip, Mary Barker, Wendy Lawrence, Cyrus Cooper and Sian Robinson \\ MRC Epidemiology Resource Centre, Southampton, UK
}

The Southampton Women's Survey (SWS) has measured the diet, body composition, physical activity and social circumstances of a large group of non-pregnant women aged 20-34 years living in the city of Southampton, UK. Diet over the 3 months before interview was assessed using an administered FFQ that records consumption of 100 food items. Principal component analysis was used to define dietary patterns. Women with high scores on the first component had diets that were in line with recommendations from the UK Department of Health and other agencies ${ }^{(1,2)}$. This pattern was termed the 'prudent' dietary pattern and was found to be strongly positively associated with educational attainment ${ }^{(3)}$.

Since an individual's score for the prudent dietary pattern is driven by the frequency of consumption of a limited number of food items that characterise the pattern, it was investigated whether assessing only consumption of these discriminating foods would generate a meaningful prudent diet score. Using an FFQ with a reduced number of items would be advantageous in terms of time and resources required when conducting a large survey. The twenty items with the greatest contribution by magnitude to the prudent diet score were (with direction indicated as ' + ' or ' - ') chips and roast potatoes $(-)$, peppers $(+)$, tomatoes $(+)$, meat pies $(-)$, vegetable dishes $(+)$, courgettes $(+)$, sausages $(-)$, gravy $(-)$, green salad $(+)$, sugar $(-)$, wholemeal bread $(+)$, white bread $(-)$, onion $(+)$, vegetarian foods $(+)$, pasta $(+)$, Yorkshire puddings and savoury pancakes $(-)$, crisps $(-)$, beef $(-)$, spinach $(+)$ and full-fat milk $(-)$.

A shortened twenty-item prudent diet score was calculated for the first 6125 SWS participants using the responses and coefficients for just the twenty foods noted earlier. The association between the full 100-item prudent diet score and this shortened twenty-item score was very strong $(r$ 0.94, $P<0.0001)$. The association between educational qualifications and the shortened twenty-item prudent diet score was also strong (Fig. 1).

The correlation between the two prudent diet scores within the SWS may be unrealistically optimistic since the choice of twenty food items was based on those items seen to contribute most strongly to the 100-item prudent diet score. To explore this issue further a twentyitem version of the FFQ was administered to assess diet in 378 women in Southampton attending SureStart Children's Centres (the Nutrition and Wellbeing Study). Using the coefficients from the SWS a prudent diet score was calculated for these women. The variation in scores in the Nutrition and Wellbeing Study was as great as that within the SWS and there was no difference in mean scores between the two studies $(P=0.85)$. The association with educational qualifications was also very similar to that in the SWS (Fig. 2).

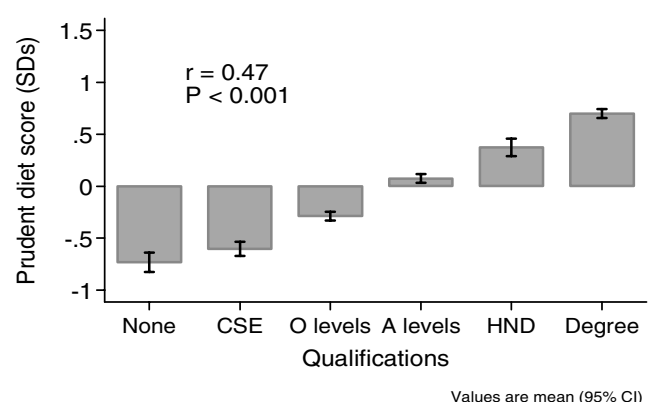

Figure 1. SWS.

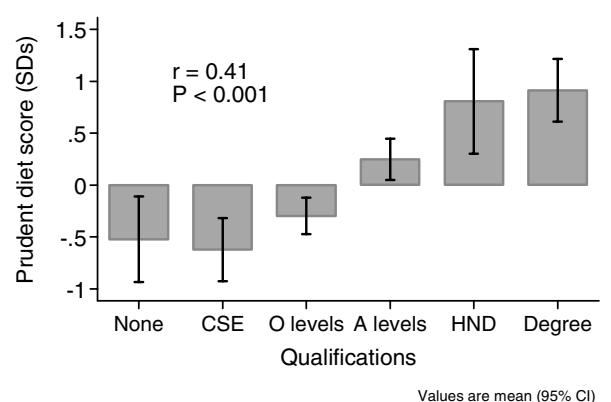

Figure 2. Nutrition and Wellbeing study.

It is concluded that the shortened FFQ enabled the calculation of a meaningful prudent dietary score without the need to administer the longer 100-item FFQ. This score indicated compliance with healthy eating messages and captured variation in women's diets, even within a more limited social setting. The associations with educational qualifications were consistent between the two studies, indicating the ability to describe a pattern that is likely to illustrate an important characteristic of women's dietary choices.

1. Department of Health (1994) Nutritional Aspects of Cardiovascular Disease. Report on Health and Social Subjects no. 46. London: H. M. Stationery Office.

2. Department of Health (1998) Nutritional Aspects of the Development of Cancer. Report on Health and Social Subjects no. 48. London: The Stationery Office.

3. Robinson SM, Crozier SR, Borland SE, Hammond J, Barker DJP \& Inskip HM (2004) Eur J Clin Nutr 58, 1174-1180. 